\title{
Intermédialités
}

Histoire et théorie des arts, des lettres et des techniques

Intermediality

History and Theory of the Arts, Literature and Technologies

\section{Le chef invité de Tarragone}

\section{Sarah Rocheville}

Numéro 5, printemps 2005

Transmettre

Transmitting

URI : https://id.erudit.org/iderudit/1005497ar

DOI : https://doi.org/10.7202/1005497ar

Aller au sommaire du numéro

\section{Éditeur(s)}

Centre de recherche sur l'intermédialité

ISSN

1705-8546 (imprimé)

1920-3136 (numérique)

Découvrir la revue

Citer cet article

Rocheville, S. (2005). Le chef invité de Tarragone. Intermédialités /

Intermediality, (5), 163-172. https://doi.org/10.7202/1005497ar
Résumé de l'article

Comment penser le rôle du chef d'orchestre contemporain? Que nous fait entendre la nouvelle et étonnante "gestique " musicale et en quoi cette nouvelle visibilité annonce-t-elle le déplacement moderne de l'autorité qui, traditionnellement, se fondait sur une sonorité phénoménale (la musique entendue) et qui semble aujourd'hui se préoccuper surtout du malaise sonore contenu dans la partition écrite? Partant d'une expérience d'écoute qui eut lieu lors d'un concert en juillet 2004, cet article propose de réfléchir au différend qui médiatise la vision du chef et l'oreille du public. Si le chef se donne encore à voir comme un transmetteur (en ce qu'il reconduit), sa transmission paraît toutefois négative, passive, coupée de tout passage à l'acte, bref, possible peut-être uniquement dans une sphère de puissance qu'il est important d'interroger à l'heure où les techniques électroacoustiques semblent faire se résorber le brouillage qui fait pourtant la fortune du concert.
Ce document est protégé par la loi sur le droit d'auteur. L'utilisation des services d’Érudit (y compris la reproduction) est assujettie à sa politique d'utilisation que vous pouvez consulter en ligne.

https://apropos.erudit.org/fr/usagers/politique-dutilisation/ 


\title{
Le chef invité de Tarragone
}

\author{
Sarah Rocheville
}

E

n juillet dernier avait lieu à Tarragone un concert donné par l'orchestre là sur les murs de la ville indiquaient aux touristes et aux mélomanes le chemin pour se rendre à pied au Teatro Romano. À 22 hoo, l'orchestre ouvrirait la soirée avec la Deuxième symphonie de Schumann, on entendrait ensuite les Tableaux d'une exposition de Moussorgski; après une pause, on terminerait avec la Symphonie $n^{\circ} 5$ de Chostakovitch. Un vin d'honneur serait servi dans la nuit. «Venez en grand nombre», pouvait-on lire sur l'affiche, «il y aura un chef invité.»

Construit au ${ }^{\text {er }}$ siècle av. J.-C. et restauré à la fin du XIX ${ }^{\mathrm{e}}$ siècle, le théâtre romain de Tarragone a conservé sa fosse et ses gradins (cavea), sa scène et, vraisemblablement, son orchestre. Ce soir-là, plus d'une centaine de personnes chuchotaient entre elles le nom du chef invité: le concert allait commencer. À 22 ho5, les instrumentistes se turent, le chef invité fit son apparition, quelques projecteurs s'éteignirent malgré l'obscurité naissante. Le public applaudit, se racla la gorge ; certains se mouchèrent bruyamment, d'autres sortirent un bonbon digestif d'une enveloppe craquante. Une dame éleva la voix pour faire taire un enfant, un homme confia une information pressante à l'oreille de son voisin, on sentait la fébrilité du technicien vérifiant en douce l'effet de sa nouvelle console. Chacun guettait un signe du chef. Il n'était de passage en Catalogne que pour trois jours, il serra la main du premier violon. Mais avant que le public n'entende les premiers accords de Schumann, une chose étrange se produisit: le chef invité s'agita sur la scène, il exécuta quelques pas de danse - se dandina - , sa baguette rebondit dans l'air, précise et luisante. On comprit que le chef entendait quelque chose qu'on ne saisissait pas. On espéra que les musiciens de l'orchestre aient saisi cette chose. Le chef gesticula encore, il respira 
d'un coup sec et enfin, les sons entrèrent dans la nuit, solidaires, retenus un moment dans l'enceinte de l'amphithéâtre romain.

Mis à part l'effet comique que crée pareil sautillement de la part d'un chef invité (observant avec Bergson qu'un mouvement mécanique plaqué sur du vivant devient risible ${ }^{1}$ ), la scène peut étonner. Alors que le rôle du chef exige habituellement de battre la mesure, de «conduire» la musique et les musiciens ou de synchroniser les différentes partitions ${ }^{2}$, il paraissait clair que la "gestique » nouvelle du chef invité, au contraire, visait à brusquer la codification traditionnelle musicale, à donner la préséance au geste sur le son (et par là, interroger l'autorité du son), à refuser la tyrannie de «l'horloge temporelle ${ }^{3}$ », à créer,

1. «Supposons qu'on appelle notre attention sur [la] matérialité du corps. Supposons qu'au lieu de participer de la légèreté du principe qui l'anime, le corps ne soit plus à nos yeux qu'une enveloppe lourde et embarrassante, lest importun qui retient à terre une âme impatiente de quitter le sol. Alors le corps deviendra pour l'âme ce que le vêtement était tout à l'heure pour le corps lui-même, une matière inerte posée sur une énergie vivante. Et l'impression du comique se produira dès que nous aurons le sentiment net de cette superposition. » (Henri Bergson, Le rire. Essais sur la signification du comique, Paris, Presses universitaires de France, coll. «Quadrige», 1989 [1900], p. 38)

2. Cette pratique obéit aux principes de direction énoncés par Hector Berlioz dans son Grand traité d'instrumentation et d'orchestration modernes, Peter Bloom (dir.), Kassel, Bärenreiter, $2004[1844,1855]$. Pour une histoire de la direction d'orchestre, de sa genèse à ses pratiques actuelles, voir les ouvrages de Georges Liébert, Ni empereur ni roi, chef d'orchestre, Paris, Éditions Gallimard, coll. «Découvertes», 1990 et Georges Liébert (éd.), L'art du chef d'orchestre: un choix de textes, Paris, Éditions Hachette, coll. «Pluriel», 1988. Voir également le dossier spécial «À quoi sert le chef d’orchestre? », Classica, n 49 , février 2003. Pour situer les enjeux musicaux contemporains, on se reportera à l'ouvrage de Bernard Sève, L'altération musicale, Paris, Éditions du Seuil, coll. «Poétique », 2002 ; à Pierre Boulez, Conversations de Pierre Boulez sur la direction d'orchestre (avec Jean Vermeil), Paris, Éditions Plume, 1989; Hermann Scherchen, La direction d'orchestre, Arles, Éditions Actes Sud, 1986 [1929]; Élisabeth Bernard, Le chef d'orchestre, Paris, Éditions La Découverte, 1989; et Elliot W. Galkin, A History of Orchestral Conducting in the Theory and the Practice, New York, Pendragon Press, 1988.

3. Selon l'expression usuelle du discours pédagogique réservé à la direction d'orchestre: «Ce qu'il faut retenir, c'est qu'en direction d'orchestre, cette maîtrise de l'horloge temporelle d'une part, et d'autre part cette responsabilité sur l'interprétation de chaque membre de l'orchestre peuvent être utilisées de façon extrêmement différentes, il peut arriver qu'un orchestre habituellement bon sonne bien mal s'il est mal dirigé, et au contraire qu'un orchestre amateur ou de seconde catégorie se trouve magnifié par la 
somme toute, un brouillage dans la circulation partition-chef-auditeur. L'observateur le moins averti voyait que le chef dirigeait Schumann comme s'il s'agissait d'une pièce contemporaine, dont la performance paraît souvent désordonnée, savante et instigatrice de perplexité. Le chef poussait de petits cris en dirigeant, tapait du pied; certains prétendirent qu'il riait au troisième tableau de Moussorgski. Il battait une mesure irrégulière à l'aide de ses talons; à deux reprises, il baissa les bras, cessant vraisemblablement de diriger: le chef faisait contrepoids à la musique entendue, ou plutôt, sa propre écoute (dont on ne pouvait douter) se montrait telle une activité parallèle, déphasée, arythmique. Le chef spectacularisait le hiatus entre ce qu'il entendait et ce qui était perçu; s'il mettait en scène le raccord entre la partition et le public, il le faisait sur le mode du faux-raccord et de la dissonance.

Pour le spectateur, l'affaire ne pouvait en rester là. Si le chef avait paru peu se soucier des conventions élémentaires - l'on avait quant à soi réservé les froissements de papier, toussotements, commentaires et élans de toutes sortes pour l'entracte et la fin du concert - , il y avait eu pire. Le chef avait donné à voir une musique différente de celle que l'on pouvait entendre, et cette visibilité à laquelle il s'était livré le révélait lui-même comme un médium négatif: le chef mettait au jour l'écart entre les multiples médiations du concert, et cette nouvelle transparence, au lieu de favoriser le circuit communicationnel, participait de l'échec de la transmission. En ce sens, le chef de Tarragone assumait un malaise moderne dès lors qu'il se présentait uniquement sous la forme d'un reste, d'un obstacle qui semblait mener à une médialité nouvelle par rapport à la tradition. S’il ne cherchait visiblement pas à créer, par sa gestique, une circulation immédiate entre la partition et la musique, il s'agissait peut-être pour lui de configurer la disparition ou la mort de la possibilité de toute immédiation dans la musique. Sans doute le chef avait-il chorégraphié ce qui appartenait à une autre temporalité, c'est-à-dire la musique émanant de la partition seule, sans égard aux conditions de possibilités réelles de cette musique. Il avait suivi du geste la virtualité musicale propre à la lecture de la partition, et cette virtualité ne pouvait se partager, sauf, peut-être, en se montrant ou en se transposant sur le mode visible.

présence et le savoir d'un chef d'orchestre [sic] », Raphaël Sanchez, «Virtuosité et management ", De l'art de la direction d'orchestre, ICAD (Institut des cadres dirigeants), Paris, Conférence du 30 mai 2000. 
En grec, l'orkêstêr, le « danseur», correspond au verbe orkheisthai, «danser» - le plus souvent en groupe ${ }^{4}$. À Rome, l'orchestre désignait la partie du théâtre réservée aux sénateurs; au XVII ${ }^{\mathrm{e}}$ siècle, cette partie, toujours contiguë à la scène, s'est transformée en fosse de contre-bas et les sénateurs qui l'occupaient ont fait place à des instrumentistes. À Tarragone, les musiciens ont joué dans la fosse de l'amphithéâtre romain et le chef-danseur invité a contré la gestuelle attendue (la chorégraphie d'ensemble), faisant fi du public (qui a l'habitude de voir se redoubler l'intention musicale dans les gestes du maestro) et aussi, d'une certaine façon, des instrumentistes. Alors que dans la tradition classique, on pouvait s'attendre au déploiement d'une harmonie imitative entre le chef et sa partition, rien de tel ne s'était produit ce soir-là. Bien sûr, le redoublement habituel du geste signifiant, même s’il avait été visé par le chef, n'aurait pu s'accomplir. Car le redoublement moderne enlève sa substance à la chose échangée, faisant se déplacer le rapport différence et répétition vers une répétition qui toujours diffère, incapable de revenir au même. Sachant que le retour de la partition vers elle-même (sa répétition), grâce à la technique du chef, n'était plus possible dans ce changement de paradigme, la répétition à laquelle se livrait le chef renvoyait à une origine toujours à venir et non plus à recouvrer.

Sans doute cette technique de direction a-t-elle tout à voir avec un fairesigne qui susciterait davantage la faconde du phénoménologue que celle du sémioticien. Si la performance du chef s'intégrait au projet de Pierre Boulez qui, dès les années 1950, a proposé une direction d'orchestre assumant un ensemble de signes formant un code que seuls les instrumentistes pouvaient comprendre - puisque le code repose sur une décentralisation du geste et que seuls les lecteurs de la partition ont accès à la possibilité du centre de référence - - il a semblé, ce soir-là de juillet, qu'au lieu de faire des signes visant à mettre en évidence, au moyen de résonances plus ou moins appuyées, les structures formelles des pièces, le chef invité de Tarragone a exécuté un fairesigne, et dans cette exécution, a sacrifié les avantages du signe et du code. Ce sacrifice a néanmoins mis au jour une technique. En se séparant de l'origine textuelle, le chef en a montré la technique et par ce fait, a donné lieu à une

4. Verbe dont l'étymologie obscure se rapproche du sanskrit rghayati, «trembler», "se déchaîner ». Pour les remarques étymologiques, on se reportera à Alain Rey, Dictionnaire historique de la langue française II, Paris, Le Robert, 1992, p. 1376, à l'entrée «orchestre». 
esthétisation de la pratique. La gestique du chef d'orchestre, loin d'apparaître comme une ornementation, se fit pratique en tant que telle, renversant par là sa fonction habituelle. On comprend alors qu'au lieu d'essayer de cacher les maladresses et les disgrâces de la technique musicale d'orchestre, il s'agissait au contraire de les montrer, quitte à y voir le risible ou la mécanique (la technologie) montrée dans sa pure fonctionnalité. Les gestes du chef n’étaient pas destinés aux instrumentistes, ils répondaient à l'appel «du texte », de la partition, et relançaient, dès leur exécution, l'échange au cours duquel l'art pouvait surgir. Il n'y eut pas de signes compris au sens de repères, il y eut un faire-signe (une action, une gestuelle, une energeia) dont l'enjeu découvrait une ontologie de l'expression musicale.

Tout se passa donc comme si le concert n'avait eu lieu (n'avait de lieu) que dans l'échange reliant le chef et la partition (à son tour partagée ou partionnée). À aucun moment le chef ne leva les yeux de son large lutrin. Ainsi son rôle était-il loin de s'apparenter à celui d'un metteur en scène, d'un répétiteur, d'un souffleur, d'un régulateur rythmique ou d'un rhétoriqueur magnifiant la musique auprès du public. Le chef avait été invité à interpréter les œuvres du programme, personne ne s'étonna du fait qu'il se présentât, somme toute, en soliste. «Pauvres compositeurs! », avait prévenu Berlioz, «sachez vous conduire et bien vous conduire! (avec ou sans calembour) car le plus dangereux de vos interprètes, c'est le chef d'orchestre, ne l'oubliez pas5.» Mais comment voir dans le chef d'orchestre un musicien, sous quels motifs lui accorder le privilège de l'interprétation, lui qui ne joue, ne frotte, ne souffle, ni ne fait sonner une seule note? Comment concéder un tel pouvoir à celui dont l'activité se limite à une lecture - anticipée, décalée, inappropriée de surcroît?

Il est souvent arrivé à Mozart de diriger son orchestre en jouant au clavier. Cette façon de faire jouer (ou de faire entendre) en jouant soi-même reprend la technique médiévale des maîtres de chapelle (kapellmeister) qui battaient fortement la mesure avec un long bâton ${ }^{6}$. Fondant sa légitimité sur la maîtrise

5. Hector Berlioz, Mémoires. Comprenant ses voyages en Italie, en Allemagne, en Russie et en Angleterre, 1803-1865, Paris, Éditions Librairie nouvelle, 1999 (manuscrit numérisé à cette adresse électronique : <http ://hector.ucdavis.edu/Berliozzo03/Memoires/ MemoooTP.htm>).

6. Pour des précisions sur le rôle du Kapellmeister, notamment sur sa contribution essentielle, jusqu'au XvIII ${ }^{\mathrm{e}}$ siècle, à tous les aspects de l'orchestre, incluant la composition, le travail de copie, les répétitions et les performances, voir l'article d'Antonio Bowen, "The rise of conducting", dans Antonio Bowen (dir.), Conducting, Cambridge, Cambridge University Press, 2003, p. 93-113. 
d'une rhétorique qui privilégie l'actio, c'est-à-dire l'art de bien dire le texte musical (le prononcer à voix haute - le solfier — , le chanter ou le jouer), l'art du chef d'orchestre se mesurait à sa capacité à donner vie au silence de la lettre écrite (la partition) en lui insufflant une dimension sonore. Non seulement ce chef incarnait-il le premier acteur de la musique, mais son action se démultipliait dans les gestes des musiciens de l'orchestre qui amplifiaient son intention de façon pléonastique (allant jusqu'à appréhender l'orchestre, dans certains cas, comme une manifestation stylistique, une ornementation du battement central). Encore aujourd'hui, il est courant de voir, dans les ensembles de musique de chambre, le premier violon (même s'il demeure assis à gauche) donner le ton, l'esprit et le tempo avant que tous ne commencent à jouer. Dans certaines chorales, le directeur chante, dos à la foule, en articulant exagérément les syllabes; parfois même, il chante plus fort que les autres. Rien de tel pour le chef invité de Tarragone, pas de transmission du sentiment musical en vertu d'une pédagogie imitative, pas de prolongement impérialiste du geste dans le son. Il y eut au contraire une coupure entre l'orchestre (pluriel, sonore, composé de partitions) et le chef (singulier, sans instrument, n'ayant accès qu'au tout), une irréconciliation qui n’a cessé, tout au long du concert, de s'aiguillonner.

Car tout comme il est impossible à un individu d'entendre sa propre voix «de l'extérieur», le musicien d'un orchestre - contrairement au chef - ne peut percevoir le son (et par là, la musique) qu'il crée. D’une part, sa position « interne » l'empêche d'entendre l'ensemble de l'orchestre; d'autre part, sa partition (écrite) est frappée en «surimpression » par rapport à celles de ses collègues et auxquelles il n'a pas accès. La partie, pour le musicien d'orchestre, devient plus grande que le tout et cette disproportion non seulement corrompt sa perception, mais modifie son jugement, c'est-à-dire son écoute.

Le problème n'est pas nouveau. Il y a, dans l'exécution d'une partition, le sacrifice de la totalité (la musique entendue «idéalement») compensé par l'exercice d'un pouvoir - les musiciens de Tarragone ont joué, contrairement au chef invité, et c'est là leur pouvoir. Mais cette fois, le chef ne s'est pas présenté comme l'incarnation de l'idée musicale (idée entendue dans un sens platonicien comme intuition de la totalité), il n'a prétendu assumer aucune posture centrale ou irradiante: le chef a fait se déplacer l'autorité vers une instance beaucoup plus complexe, celle de la partition d'ensemble (expression antithétique) qui elle, recompose la totalité et détient, en ce sens, le savoir, c'est-à-dire la musique comme puissance réalisée lors de la seule lecture. Seul le chef 
invité avait accès à cette partition d'ensemble. Et c'est bien avec elle qu'il parlait, riait, chantait ou dialoguait, bref, c'est à elle qu'il avait répondu lors de l'invitation.

C'est là que s'est présentée, ce soir-là, la tension nécessaire au surgissement de la musique et par extension, de l'art. Il y eut une séparation radicale entre la musique écrite - dont rendait compte simultanément le chef en se dandinant - , c'est-à-dire lue, et la musique qui a pour essence d'être sonore, proférée, manifestée. On perçut un retard entre la vision du chef et l'audition du public, il y eut un différend entre l'œil et l'oreille et le chef, loin de s'y soustraire, en exploitait les limites. «Introduire du retard dans l'immédiat: c'est la musique (ou la mémoire) et c'est pourquoi mnèmosynè et musica sont les mêmes ${ }^{7}$. Peut-être pourrait-on comprendre ce retard qui s'induit dans le décalage que le chef manifeste comme le retard de l'étant sur l'être. D'une certaine façon, l'étant deviendrait assez transparent pour montrer ses mécanismes, mais il ne serait pas pour autant capable de remédier à son inadéquation. Car de quelle façon pourrait-on franchir le pas entre la certitude sensible et l'immédiat? C'est bien toujours par le médium, c'est-à-dire le retard, la mémoire, la tradition entendue comme ensemble de pratiques. Voilà que se découvre un ancrage ontologique à la question de l'intermédialité. Si la mémoire introduit le décalage, elle est aussi la muse de la technique ${ }^{8}$.

Sans doute l'attention du chef d'orchestre se tourne-t-elle vers ce caractère musical qui rend sensible le retard, la non-coïncidence. Si, depuis la souple notation mélodique du chant grégorien jusqu'à la mise en place d'un système normatif instauré à la Renaissance, la partition musicale a surtout servi de support ou d'aide-mémoire aux musiciens et aux chefs; si la note muette sur le papier, lettre errante dont la matérialisation ne se résorbait que dans sa prononciation audible, a eu besoin, jusqu'au milieu du $\mathrm{xx}^{\mathrm{e}}$ siècle, de l'actio pour donner vie à la musique, force est de reconnaître que le système de composition contemporain a tenté de rejouer cette soumission du noté à l'entendu en proposant des modèles d'écriture musicale qui permettent, à la seule lecture

7. Pascal Quignard, La haine de la musique, Paris, Éditions Gallimard, coll. «Folio », 1997 [1996], p. 33.

8. Technique distinguée de technologie. Voir à ce propos Giorgio Agamben, Moyens sans fins: notes sur la politique, Paris, Payot et Rivages, coll. «Bibliothèque Rivages", 2002 [1995]. Sur Mnémosyne et la technique, voir la présentation d’Éric Méchoulan, «Aimer - la technique », Intermédialités, $n^{\circ}$ 4, «Aimer», automne 2004, p. 9-17. 
- c'est-à-dire «à la vue» de la partition —, d'entendre une musique irréductible à sa profération. Se redéfinit du coup le rôle du chef d'orchestre qui, eu égard au répertoire qu'il interprète, ne sert plus à diriger l'orchestre, mais plutôt à en reconduire la manifestation musicale vers une autre musique (inouïe) qui la précède.

Notes ininterprétables, sons non sonores, signes qui sont inscrits pour la pure beauté de l'écriture. Je propose d'appeler «notes inouïes» ces sons écrits injouables qui font penser à ce que les grammairiens nomment les «consonnes ineffables» (le p dans sept $)^{9}$.

De là une série de conséquences qui bousculent et réinscrivent le rôle du chef d'orchestre dans une modernité scriptocentrique. À la fois hors du groupe des instrumentistes et au centre des musiciens, le chef assume une responsabilité délicate puisqu'il se place à la fois au centre et en périphérie du jeu musical: il fait du hors-jeu le centre du jeu. Mais tout comme l'araignée ${ }^{10}$, tapie à l'extérieur de sa toile, constitue néanmoins le centre mobile et caché d'une toile, c'est bien la note écrite, appelante et inouïe, qui fait office de centre de la manifestation musicale et non le chef d'orchestre qui, visible, passif (qui subit) et passionné (souffrant de sa non-coïncidence), ne fait que reconduire la note autoritaire, noire et impuissante vers une manifestation audible (ouïe), c'est-à-dire partitionnée et puissante. Le chef se donne donc à voir comme la visibilité même du scriptible, comme la puissance cherche un acte, au sens aristotélicien :

Rien ne ressemble davantage à cela que la condition qu'Aristote, en l'opposant à l'acte, définit comme "puissance». La puissance est d'une part, potentia passiva, passivité, passion pure et virtuellement infinie, d'autre part potentia activa, tension irréductible vers l'accomplissement, passage à l'acte ${ }^{11}$.

9. Pascal Quignard, La haine de la musique, p. 71.

10. L'image de l'araignée velue, laide et disgracieuse est adéquate à celle du chef d'orchestre moderne qui rend visible sa disgrâce en gesticulant dans son habit à basques en queue de morue. Tout comme chez Pascal, l'ange se transforme en bête, cette disgrâce est rendue visible par la mise en évidence de la technique. Il y a là, dans cette disgrâce mise à nue, le procès d'une rhétorique qui, parfaitement exécutée, transgresserait son ordre linguistique pour éventuellement se transformer en grâce.

11. Giorgio Agamben, Idée de la prose, trad. Gérard Macé, Paris, Éditions Christian Bourgois, coll. «Détroits», 1998 [1988], p. 46. 
Le chef se donne à voir comme un transmetteur (en ce qu'il reconduit), mais sa transmission est ainsi négative, puissante, elle procède d'un "céder", comme l'on cède un droit ou un bien. En cédant son autorité à la note inouïe (écrite), le chef devient une instance en surplus, dérangeante, parfois grotesque et même risible, bref, incapable de se rendre digne de sa visibilité. On comprend alors que le chef de Tarragone «spectacularisait» le caractère singulier de sa fonction qui appartient au règne du «trop », du «après » ou du «avant»; il avait rompu la fluidité de l'ineffable en permettant à la musique de se manifester. Sans doute son rire et sa danse participèrent-ils de ce même excès. Sans doute le chef d'orchestre occupe-t-il aussi, en ce sens, une fonction analogue à celle de la langue pour Pascal Quignard.

Lingua accroît du «hors", du «après-coup», de l'absence, du discontinu, de la mort, de la vision binaire, du couple, de l'intervalle, du duel, du sexe, de la lutte. De même que la négation aux yeux du linguiste ne retranche rien: elle ajoute à la phrase positive les marques de ce qui la nie ${ }^{12}$.

Comme la négation qui ne retranche rien, la présence visible et, en un sens, surannée du chef d'orchestre ajoute à la musique ce qui la sépare d'ellemême. Si elle n'enlève rien, cette négation ajoute du moins une séparation dont la musique ne peut faire l'économie pour advenir dans sa phénoménalité. Certes le chef d'orchestre demeure un vecteur lors du spectacle, mais sa présence fait refluer la circulation musicale en manifestant une sorte de reste, de scorie, de fossile d'un orkhêstra qui s'agite dans la mémoire de l'interprète et de l'auditeur. On peut se demander si ce rôle de transmission n'est pas appelé à disparaître, comme Glenn Gould le présageait dans une semonce de la pratique symphonique contemporaine.

La direction d'orchestre de l'avenir sera assurée par un synthétiseur d'inspiration hypnotisante en circuit fermé, une sorte de pot-pourri démocratiquement programmé des idées et idéaux des musiciens d'orchestre, des managers et des critiques, enfin rassemblés dans une vision unifiée des choses. Sa conception convaincante du répertoire sera transmise aux musiciens d'orchestre à l'intérieur de leurs studios qui seront bien évidemment le lieu exclusif des répétitions et des exécutions, l'assemblage des sons étant assuré par des commandes programmées à partir d'une série de spécifications a priori définies. Ainsi le musicien d'orchestre sera-t-il en mesure

12. Pascal Quignard, La haine de la musique, p. 33. La discontinuité du sexe suspend le geste de l'accouplement et esthétise sa pratique. La sécrétion, ainsi, dévoile le secret et rend problématique le mystère de la langue. 
de franchir des pas de géant en direction d'un avenir automatisé. Il pourra jouer tout en restant chez lui, oublier ses problèmes de voiture, s'épargner les services d'une baby-sitter et envoyer sa femme au travail ${ }^{13}$.

Quittant une présence sensible et visible, la direction d'orchestre tendrait à se concentrer sur sa pure fonctionnalité et s'essaierait à contenir toute forme de reste ou de débordement. Ce qui distingue l'action de transmettre que peut exercer un chef d'orchestre de la pure fonction qu'il devient dans un système sémiotique tient justement dans ce brouillage du circuit de la communication qui semble appelé à disparaître. Lorsqu'il y a transmission, quelque chose se perd et se donne, quelque chose, justement, est retenu - dans la mémoire mais aussi dans la fluidité du circuit qui va, par exemple, de la partition vers la totalité de la pièce musicale. Si cette retenue disparaît, si le dandinement rieur du chef se fige, le relais, même inadéquat, n'est plus possible. Pourquoi cela? S'il n'y avait plus de relais, mais au contraire une transmission directe et immédiate, cela équivaudrait-il à une absence de médium? Est-ce dire qu'une direction qui exacerberait la technologie (déchéance de la technique en technologie) empêcherait la transmission par laquelle l'art est possible? Au contraire, il s'agit de repenser le rôle de la direction d'orchestre en fonction de cette technique qui n'avait d'ailleurs jamais été occultée. Des manifestations antiques chorégraphiées aux essais de musique électroacoustique en temps réel, il n'y a qu'un déplacement des endroits de brouillage - donnant lieu à de multiples transferts qu'il s'agit d'interroger - et non un changement d'enjeu. En donnant à l'opérateur (opus) acousticien le rôle de pur décideur, on a confondu dans une seule et même action interprétation et composition et, par le fait même, remis en cause la pertinence du brouillage qu'effectue le chef dans la transmission. Il n'en demeure pas moins que la technique nécessite, malgré tout et peut-être malgré elle, une certaine forme de transmission (excédant par là sa pure fonction) dans la mesure où la seule façon pour le compositeur-interprète électroacousticien de faire se résorber le brouillage, le reste ou la négativité dont son activité ne peut faire l'économie demeure l'arbitraire, c'est-à-dire le pouvoir de la décision, de ce qui relie en coupant.

13. Herbert von Hochmeister [Glenn Gould], «Of Time and Time Beaters », Contrepoint à la ligne, cité par Peter Szendy dans son très éclairant article consacré à l'évolution de la musique électroacoustique, «De la harpe éolienne à la "toile": fragments d'une généalogie portative », Traverses, $n^{\circ}$ 1, Éditions du Centre Georges Pompidou, juillet 1996, <http://www2.centrepompidou.fr/traverses/numero1/textes/t-szen.htm>. 